\title{
Hermeneutik der Gleichnisse Jesu
}

\author{
Methodische Neuansätze zum Verstehen urchristlicher Parabeltexte \\ Hrsg. v. Ruben Zimmermann unter Mitarb. v. Gabi Kern
}

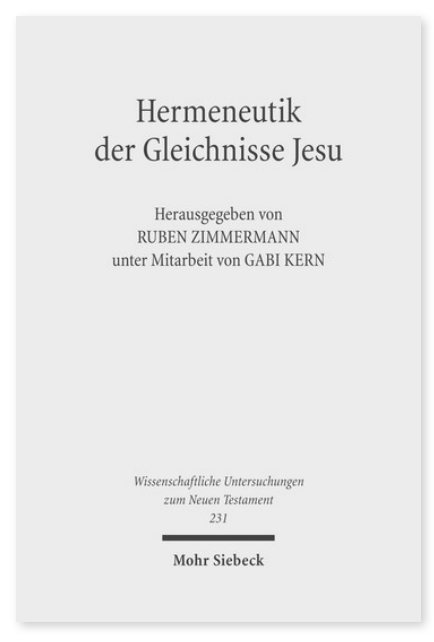

2008; unveränderte Studienausgabe: 2011. XII, 688 Seiten. WUNT I 231

ISBN 978-3-16-151520-0 DOI 10.1628/978-3-16-151520-0 eBook PDF $94,00 €$

ISBN 978-3-16-150850-9 fadengeheftete Broschur $94,00 €$
Gleichnisse sind Rätseltexte. Sie sind nicht klar und eindeutig, sondern setzen einen Prozess der Deutung in Gang, der je nach Perspektive unterschiedlich ausfallen kann. So gibt es nicht nur eine richtige Interpretation eines Gleichnisses, sondern vielmehr unterschiedliche Verstehensmöglichkeiten, die je und je neu gefunden werden müssen. In ihrer bewusst kalkulierten Deutungsoffenheit machen die Gleichnisse die Reflexion der Verstehensvoraussetzungen und methoden unerlässlich, sie erfordern Hermeneutik.

Der vorliegende Sammelband stellt sich dieser Herausforderung und dokumentiert dabei zugleich hermeneutische Weichenstellungen, die innerhalb des Kompendiums der Gleichnisse Jesu (hg. v. R. Zimmermann, 2007, Gütersloher Verlagshaus) leitend wurden.

Im Einzelnen werden historische (z.B. sozialgeschichtliche), sprachwissenschaftliche (z.B. gattungstheoretische) sowie rezeptionsästhetische (z.B. semiotische) Ansätze der Gleichnisdeutung vorgestellt und diskutiert. Dabei wird immer wieder der Dialog mit der amerikanischen Gleichnisforschung gesucht.

Neben der Divergenz der Verstehenswege sollen aber auch Schnittstellen und mögliche Verknüpfungen unterschiedlicher Perspektiven im Horizont einer 'integrativen Gleichnishermeneutik' sichtbar werden.

\section{Inhaltsübersicht}

Ruben Zimmermann: Im Spielraum des Verstehens. Chancen einer integrativen Gleichnishermeneutik - Uta Poplutz: Parabelauslegung im Kompendium der Gleichnisse Jesu. Reflexion der Methodenschritte und exemplarische Exegese von Joh 3,29f.

\section{Historische und sozialgeschichtliche Perspektiven}

Ruben Zimmermann: Gleichnisse als Medien der Jesuserinnerung. Die Historizität der Jesusparabeln im Horizont der Gedächtnisforschung - Karl-Heinrich Ostmeyer: Gleichnisse. Quelle des Verständnisses der Umwelt Jesu? Umwelt. Quelle des Verständnisses der Gleichnisse Jesu? - Luise Schottroff: Sozialgeschichtliche Gleichnisauslegung. Überlegungen zu einer nichtdualistischen Gleichnistheorie - Warren Carter: Matthew's Gospel, Rome's Empire, and the Parable of the Mustard Seed (Matt 13:31-32) - Charles W. Hedrick: Survivors of the Crucifixion. Searching for Profiles in the Parables - Klyne Snodgrass: Stories with Prophetic Intent. The Contextualization of Jesus' Parables

II. Traditions- und redaktionsgeschichtliche Perspektiven

Andreas Schüle: Mashal (Ivm) and the Prophetic »Parables« - Catherine Hezser: Rabbinische Gleichnisse und ihre Vergleichbarkeit mit neutestamentlichen Gleichnissen - Ruben Zimmermann: Jesus' Parables and Ancient Rhetoric. The Contributions of Aristotle and Quintilian to the Form Criticism of the Parables - Michael Labahn: Das Reich Gottes und seine performativen Abbildungen. Gleichnisse, Parabeln und Bilder als Handlungsmodelle im Dokument Q - Kurt Erlemann: Die eschatologisch-kritische Funktion der synoptischen Parabeln - Mira Stare: Gibt es Gleichnisse im Johannesevangelium? Enno Edzard Popkes: 'Das Mysterion der Botschaft Jesu'. Beobachtungen zur synoptischen Parabeltheorie und ihren Analogien im Johannesevangelium und Thomasevangelium - Clare Rothschild: Parabolh, in Hebrews III. Sprach- und literaturwissenschaftliche Perspektiven Ruben Zimmermann: Parabeln - sonst nichts! Gattungsbestimmung jenseits der Klassifikation in »Bildwort«, »Gleichnis«, »Parabel« und »Beispielerzählung« - Detlev Dormeyer: Gleichnisse als narrative und metaphorische Konstrukte - sprachliche und handlungsorientierte Aspekte - Christian Münch: Form und Referenz von Gleichnissen in synoptischen Evangelien Thomas Braun: Wenn zwischen den Zeilen ein Funke aufblitzt. Überlegungen zur Metaphorik lukanischer Gleichnisse im Anschluss an Paul Ricœur und am Beispiel von Lk 17,7-10 - Kurt Erlemann: Allegorie, Allegorese, Allegorisierung - Annette Weissenrieder: Didaktik der Bilder. Allegorie und Allegorese am Beispiel von Mk 4,3-20 - Adela Yarbro Collins: The Discourse in Parables in Mark 4

III. Rezeptionsästhetische und theologische Fragestellungen

Eckart Reinmuth: Vom Sprachereignis zum Kommunikationsereignis. Diskurstheoretische Überlegungen zu den Kontexten der Gleichnisrede Jesu - Kristina Dronsch: Auf der Suche nach der Bedeutung der Gleichnisse - semiotische Aspekte - Stefan Alkier: Himmel und Hölle. Zur Kontextualität und Referenz gleichnishafter Rede unter besonderer Berücksichtigung des Gleichnisses vom Fischnetz (Mt 13,47-50) - Stephen Curkpatrick: Parable and Vocative Word. A Dialogue with Levinas - Mary Ann Beavis: Feminist (and other) Reflections on the Woman with Seven Husbands (Mark 12:20-23): A Neglected Synoptic Parable - Tania Oldenhage: Jesus, Kafka und die Gräuel des 20. Jahrhunderts. Gleichnishermeneutik nach der Shoah - Arland J. Hultgren: Interpreting the Parables of Jesus. Giving Voice to Their Theological Significance

Ruben Zimmermann Born 1968; 1999 Dr. theol. from the University of Heidelberg; 2003 Habilitation from the LudwigMaximilians-University München; currently Professor of New Testament and Ethics at the Johannes Gutenberg-University in Mainz, Germany and research associate at the Department of Old and New Testament Studies of the University of the Free State, Bloemfontein, South Africa. https://orcid.org/0000-0002-1620-4396

Gabi Kern ist Wiss. Mitarbeiterin am Lehrstuhl für Biblische Theologie der Universität Bielefeld. 
Jetzt bestellen:

https://mohrsiebeck.com/buch/hermeneutik-der-gleichnisse-jesu-9783161515200?no_cache=1

order@mohrsiebeck.com

Telefon: +49 (0)7071-923-17

Telefax: $+49(0) 7071-51104$

Mohr Siebeck GmbH \& Co. KG

Postfach 2040

D-72010 Tübingen

info@mohrsiebeck.com

Mohr Siebeck

www.mohrsiebeck.com 